PROCEEDINGS OF THE

AMERICAN MATHEMATICAL SOCIETY

Volume 135, Number 11, November 2007, Pages 3581-3587

S 0002-9939(07)08996-4

Article electronically published on June 22, 2007

\title{
LIFTING OF THE APPROXIMATION PROPERTY FROM BANACH SPACES TO THEIR DUAL SPACES
}

\author{
EVE OJA
}

(Communicated by Jonathan M. Borwein)

\begin{abstract}
Inspired by the principle of local reflexivity, due to Lindenstrauss and Rosenthal, a new geometric property of Banach spaces, the extendable local reflexivity, was recently introduced by Rosenthal. Johnson and Oikhberg proved that the extendable local reflexivity permits lifting the bounded approximation property from Banach spaces to their dual spaces. It is not known whether the extendable local reflexivity permits lifting the approximation property. We prove that it does whenever the space is complemented in its bidual. This involves the concept of the weak bounded approximation property, introduced by Lima and Oja.
\end{abstract}

\section{INTRODUCTION}

Let $X$ be a Banach space and let $I_{X}$ denote the identity operator on $X$. If $I_{X}$ can be uniformly approximated on compact subsets of $X$ by finite-rank operators, then $X$ is said to have the approximation property. If there exists $\lambda \geq 1$ such that $I_{X}$ can be approximated by finite-rank operators of norm $\leq \lambda$, then $X$ is said to have the $\lambda$-bounded approximation property. The metric approximation property is the 1 bounded approximation property. If $X$ has the $\lambda$-bounded approximation property for some $\lambda \geq 1$, then $X$ is said to have the bounded approximation property.

Recently, a new geometric property of Banach spaces - the extendable local reflexivity - was discovered by Rosenthal and studied by Johnson, Oikhberg, and Rosenthal in 8 ] and [15]. As was shown by Johnson and Oikhberg [8], this property permits lifting the bounded approximation property from a Banach space to its dual space. Recall the relevant notions and result.

Let $X$ be a Banach space and let $1 \leq \lambda<\infty$. Following [15, we say that $X$ is $\lambda$-extendably locally reflexive if for all finite-dimensional subspaces $E \subset X^{* *}$ and $F \subset X^{*}$, and for all $\varepsilon>0$, there exists a bounded linear operator $T: X^{* *} \rightarrow X^{* *}$ such that $T(E) \subset X, x^{*}\left(T x^{* *}\right)=x^{* *}\left(x^{*}\right)$, for all $x^{* *} \in E$ and $x^{*} \in F$, and $\|T\| \leq \lambda+\varepsilon$. If $X$ is $\lambda$-extendably locally reflexive for some $\lambda \geq 1$, then $X$ is said to be extendably locally reflexive. The next theorem is proven in [8, Theorem 3.1]; for its quantized version, see [15, Theorem 3.13].

Received by the editors August 10, 2006.

2000 Mathematics Subject Classification. Primary 46B20, 46B28, 47L05.

Key words and phrases. Approximation properties, extendable local reflexivity, projective tensor product of Banach spaces.

This research was partially supported by Estonian Science Foundation Grant 5704.

(C)2007 American Mathematical Society

Reverts to public domain 28 years from publication 
Theorem 1.1 (Johnson-Oikhberg). If a Banach space $X$ is $\lambda$-extendably locally reflexive and has the $\mu$-bounded approximation property, then $X^{*}$ has the $\lambda \mu$-bounded approximation property.

By an important result due to Figiel and Johnson [4, the approximation property is strictly weaker than the bounded approximation property.

Besides Theorem 1.1, cases when bounded (in particular, metric) approximation properties can be lifted from a Banach space $X$ to its dual $X^{*}$ have been studied from the seminal paper [9], for instance, in [7, 44, 5], 10, [16]. In contrast, cases when the approximation property could be lifted from $X$ to $X^{*}$ seem to be unknown so far. In particular, it is not known whether the extendable local reflexivity permits lifting the approximation property. The purpose of the present article is to prove that it does whenever the space is complemented in its bidual.

Theorem 1.2. Let a Banach space $X$ be complemented in its bidual $X^{* *}$. If $X$ is extendably locally reflexive and has the approximation property, then $X^{*}$ has the approximation property.

The proof of Theorem 1.2 involves the weak bounded approximation property (see Section 2 for the definition). This property was recently introduced and studied by Lima and Oja in 12. In fact, in Section 3, we shall prove the following lifting theorem, which is the main result of this article.

Theorem 1.3. If a Banach space $X$ is extendably locally reflexive and has the weak bounded approximation property, then $X^{*}$ has the approximation property.

Since the approximation property and the weak bounded approximation property are equivalent for Banach spaces that are complemented in their biduals (see 12 or Theorem 2.1 below), Theorem 1.2 is immediate from Theorem 1.3. We do not know whether Theorem 1.2 could be proven in a direct way, without relying on the weak bounded approximation property.

The notation we use is standard. We consider Banach spaces over the same, either real or complex, field $\mathbb{K}$. Let $X$ and $Y$ be Banach spaces. We denote by $\mathcal{L}(X, Y)$ the Banach space of bounded linear operators from $X$ to $Y$ and by $\mathcal{F}(X, Y)$ and $\mathcal{K}(X, Y)$ its subspaces of finite-rank and compact operators. We shall always consider $X$ as a subspace of $X^{* *}$, identifying the canonical embedding $j_{X}: X \rightarrow X^{* *}$ with the identity embedding. The closed unit ball of $X$ is denoted by $B_{X}$, and the closure of a set $A \subset X$ is denoted by $\bar{A}$.

\section{THE WEAK BOUNDED APPROXIMATION PROPERTY AND AUXILIARY RESULTS}

2.1. Let $1 \leq \lambda<\infty$. Following [12, Theorem 2.4], we say that a Banach space $X$ has the weak $\lambda$-bounded approximation property if for every separable reflexive Banach space $Y$ and for every operator $T \in \mathcal{K}(X, Y)$, there exists a net $\left(S_{\alpha}\right) \subset$ $\mathcal{F}(X, X)$ such that $\sup _{\alpha}\left\|T S_{\alpha}\right\| \leq \lambda\|T\|$ and $S_{\alpha} \rightarrow I_{X}$ uniformly on compact subsets of $X$. We say that $X$ has the weak bounded approximation property if $X$ has the weak $\lambda$-bounded approximation property for some $\lambda$.

The $\lambda$-bounded approximation property implies the weak $\lambda$-bounded approximation property. This is obvious from the definitions. It is not known whether the weak $\lambda$-bounded approximation property implies the $\lambda$-bounded approximation property. It is conjectured in 12 that the weak $\lambda$-bounded and the $\lambda$-bounded approximation properties are, in general, different. If they were equivalent, then a 
long-standing open problem (see, e.g., [1, page 289]) would have a positive answer: the approximation property of (nonseparable) dual spaces would imply the metric approximation property (see Theorem 2.1 below.)

The weak bounded approximation property clearly implies the approximation property (take $T=0$ in the definition). The converse is not true in general (see 12 . or 17] for examples). The converse is true for Banach spaces that are complemented in their biduals, as proven in [12. More precisely, the following holds.

Theorem 2.1 (Lima-Oja). Let a Banach space $X$ be complemented in its bidual $X^{* *}$ by a projection $P$. If $X$ has the $A P$, then $X$ has the weak $\|P\|-B A P$.

Since our Theorem 1.2 relies on Theorem 2.1, for completeness, we shortly comment on its argument. Let $Y$ be any reflexive Banach space and let $X \widehat{\otimes} Y^{*}$ be the projective tensor product. The first step of the proof of Theorem 2.1 (see [12, Corollary 3.3]) consists in showing that $\|u\|_{\pi} \leq\|P\|\|V u\|, u \in X \widehat{\otimes} Y^{*}$, where $\|\cdot\|_{\pi}$ is the projective norm (see Section 2.2 below) and $V: X \widehat{\otimes} Y^{*} \rightarrow(\mathcal{F}(X, Y))^{*}$ is the trace mapping. This step uses canonical identifications of $X \widehat{\otimes} Y^{*}$ as the Banach space of nuclear operators $\mathcal{N}(Y, X)$ and of $(\mathcal{F}(X, Y))^{*}$ as the Banach space of integral operators $\mathcal{I}\left(Y, X^{* *}\right)$ (see [6] or, e.g., [3]). The weak $\|P\|$-bounded approximation property is then deduced in two more complicated technical steps (see [12, Theorem 3.2 and Theorem 2.4, $\left.\left.\left(\mathrm{d}^{\prime}\right) \Rightarrow\left(\mathrm{a}^{\prime}\right)\right]\right)$. Here one applies different aspects of the isometric version of the famous Davis-Figiel-Johnson-Pełczyński factorization lemma 22 due to Lima, Nygaard, and Oja [11. One also uses the description of the dual of $\mathcal{L}(X, X)$ in the topology of uniform convergence on compact subsets of $X$ (see [6] or, e.g., [14, page 31]).

2.2. Let $X$ and $Y$ be Banach spaces. Recall that the projective tensor product $X \widehat{\otimes} Y$ has a simple description due to Grothendieck [6] (see, e.g., 3, page 227]): every $u \in X \widehat{\otimes} Y$ has a representation

$$
u=\sum_{n=1}^{\infty} x_{n} \otimes y_{n} \quad \text { with } \quad \sum_{n=1}^{\infty}\left\|x_{n}\right\|\left\|y_{n}\right\|<\infty
$$

(the series is (absolutely) converging for the projective norm $\|\cdot\|_{\pi}$ ). Moreover,

$$
\|u\|_{\pi}=\inf \left\{\sum_{n=1}^{\infty}\left\|x_{n}\right\|\left\|y_{n}\right\|: u=\sum_{n=1}^{\infty} x_{n} \otimes y_{n}\right\}, u \in X \widehat{\otimes} Y,
$$

where the infimum is taken over all representations of $u$ of the form $(*)$.

The dual space of $X \widehat{\otimes} Y$ can be isometrically identified with $\mathcal{L}\left(Y, X^{*}\right)$ under the duality

$$
\left\langle A, \sum_{n=1}^{\infty} x_{n} \otimes y_{n}\right\rangle=\sum_{n=1}^{\infty}\left(A y_{n}\right)\left(x_{n}\right)
$$

(see, e.g., [3, pages 229-230]). Therefore one writes $(X \widehat{\otimes} Y)^{*}=\mathcal{L}\left(Y, X^{*}\right)$.

The proof of Theorem 1.3 below will use the following (probably well-known) auxiliary results where the weak ${ }^{*}$ topology on $\mathcal{L}\left(X, Y^{* *}\right)$ is induced by the duality with $Y^{*} \widehat{\otimes} X$.

Lemma 2.2. A Banach space $X$ has the approximation property if and only if the canonical embedding $j_{X}: X \rightarrow X^{* *}$ belongs to the weak ${ }^{*}$ closure of $\mathcal{F}(X, X)$ in $\mathcal{L}\left(X, X^{* *}\right)$. 
Proof. The approximation property of $X$ means that $I_{X}$ belongs to the closure of $\mathcal{F}(X, X)$ in the locally convex topology on $\mathcal{L}(X, X)$ of uniform convergence on compact subsets of $X$. By the identification of the dual space of the locally convex space $\mathcal{L}(X, X)$ due to Grothendieck [6, Chapter I, page 113] (see, e.g., [14, page 31]), this is equivalent to the fact that $I_{X}$ belongs to the closure of $\mathcal{F}(X, X)$ in the weak topology $\sigma\left(\mathcal{L}(X, X), X^{*} \widehat{\otimes} X\right)$. That is,

$$
I_{X} \in \overline{\mathcal{F}(X, X)}^{\sigma\left(\mathcal{L}(X, X), X^{*} \hat{\otimes} X\right)}=\mathcal{L}(X, X) \cap \overline{\mathcal{F}(X, X)}^{\sigma\left(\mathcal{L}\left(X, X^{* *}\right), X^{*} \widehat{\otimes} X\right)},
$$

where $\mathcal{L}(X, X)$ is considered as a subspace of $\mathcal{L}\left(X, X^{* *}\right)$. Since then $I_{X}$ identifies with $j_{X}$, this means that

$$
j_{X} \in \overline{\mathcal{F}(X, X)}^{\sigma\left(\mathcal{L}\left(X, X^{* *}\right), X^{*} \widehat{\otimes} X\right)} .
$$

Lemma 2.3. Let $X$ and $Y$ be Banach spaces. Then the weak ${ }^{*}$ closures of $\mathcal{F}(X, Y)$ and of $\mathcal{F}\left(X, Y^{* *}\right)$ coincide in $\mathcal{L}\left(X, Y^{* *}\right)$.

Proof. Let $A \in \mathcal{L}\left(X, Y^{* *}\right)$. We assume that

$$
A \in{\overline{\mathcal{F}\left(X, Y^{* *}\right)}}^{w^{*}} \subset \mathcal{L}\left(X, Y^{* *}\right)=\left(Y^{*} \widehat{\otimes} X\right)^{*} .
$$

This means that every $u \in Y^{*} \widehat{\otimes} X$ that vanishes on all 1-rank operators $x^{*} \otimes y^{* *} \in$ $\mathcal{F}\left(X, Y^{* *}\right)$ also vanishes on $A$.

To show that $A \in \overline{\mathcal{F}(X, Y)} w^{*}$, let us consider any $u=\sum_{n=1}^{\infty} y_{n}^{*} \otimes x_{n} \in Y^{*} \widehat{\otimes} X$ with $\sum_{n=1}^{\infty}\left\|y_{n}^{*}\right\|\left\|x_{n}\right\|<\infty$. Assume that $u$ vanishes on all 1-rank operators $x^{*} \otimes y \in$ $\mathcal{F}(X, Y)$, that is,

$$
\left\langle x^{*} \otimes y, u\right\rangle=\sum_{n=1}^{\infty} x^{*}\left(x_{n}\right) y_{n}^{*}(y)=0 \quad \forall x^{*} \in X^{*}, \forall y \in Y .
$$

But then, in $Y^{*}$,

$$
\sum_{n=1}^{\infty} x^{*}\left(x_{n}\right) y_{n}^{*}=0 \quad \forall x^{*} \in X^{*}
$$

Therefore

$$
\begin{aligned}
\left\langle x^{*} \otimes y^{* *}, u\right\rangle & =\sum_{n=1}^{\infty} x^{*}\left(x_{n}\right) y^{* *}\left(y_{n}^{*}\right) \\
& =y^{* *}\left(\sum_{n=1}^{\infty} x^{*}\left(x_{n}\right) y_{n}^{*}\right)=0 \quad \forall x^{*} \in X^{*}, \forall y^{* *} \in Y^{* *}
\end{aligned}
$$

By assumption, this implies that $\langle A, u\rangle=0$. Hence, $A \in \overline{\mathcal{F}(X, Y)}^{w^{*}}$ as desired.

\section{Proof of Theorem 1.3}

Let $X$ be a Banach space. We assume that $X$ is $\lambda$-extendably locally reflexive and has the weak $\mu$-bounded approximation property for some $\lambda$ and $\mu \geq 1$. We will prove that $X^{*}$ has the approximation property. According to Lemmas 2.2 and 2.3 , we need to show that

$$
j_{X^{*}} \in \overline{\mathcal{F}\left(X^{*}, X^{* * *}\right)}{ }^{w^{*}} \subset \mathcal{L}\left(X^{*}, X^{* * *}\right)=\left(X^{* *} \widehat{\otimes} X^{*}\right)^{*} .
$$


The idea of the proof is to construct a functional $f \in\left(X^{* *} \widehat{\otimes} X^{*}\right)^{*}$ in such a way that if $\varphi$ is the operator from $\mathcal{L}\left(X^{*}, X^{* * *}\right)$ canonically corresponding to $f$, then $\varphi \in \overline{\mathcal{F}\left(X^{*}, X^{* * *}\right)} w^{*}$ and $j_{X^{*}}=\varphi$.

Let us consider the set of all $\nu=(E, F, G, \varepsilon)$, where $E \subset X^{* *}, F \subset X^{*}$, and $G \subset X^{* *} \widehat{\otimes} X^{*}$ are finite-dimensional subspaces, and $0<\varepsilon<1$, directed in the natural way.

Since $X$ is $\lambda$-extendably locally reflexive, there exists an operator $T_{\nu} \in$ $\mathcal{L}\left(X^{* *}, X^{* *}\right)$ such that $T_{\nu}(E) \subset X, x^{* *}\left(x^{*}\right)=x^{*}\left(T_{\nu} x^{* *}\right)$ for all $x^{* *} \in E$ and $x^{*} \in F$, and $\left\|T_{\nu}\right\| \leq \lambda+\varepsilon$.

Since $B_{G}$ is a compact subset of $X^{* *} \widehat{\otimes} X^{*}$, by a well-known representation theorem due to Grothendieck [6. Chapter I, Theorem 1, pages 51-52], there exist null-sequences $\left(x_{n}^{*}\right) \subset B_{X^{*}}$ and $\left(x_{n}^{* *}\right) \subset B_{X^{* *}}$, and a subset $\left\{\left(\lambda_{n}^{u}\right)_{n=1}^{\infty}: u \in B_{G}\right\}$ of $\ell_{1}$ such that each $u \in B_{G}$ can be represented in the form

$$
u=\sum_{n=1}^{\infty} \lambda_{n}^{u} x_{n}^{* *} \otimes x_{n}^{*} \text { with } \sum_{n=1}^{\infty}\left|\lambda_{n}^{u}\right| \leq 1+\varepsilon .
$$

Let us denote by $K$ the closed absolutely convex hull in $X^{*}$ of the compact set $\left\{0, x_{1}^{*}, x_{2}^{*}, \ldots\right\}$. Then $K$ is an absolutely convex compact subset of $B_{X^{*}}$. By the isometric version of the Davis-Figiel-Johnson-Pełczyński factorization lemma [2] due to Lima, Nygaard, and Oja [11, there exists a separable reflexive Banach space $Y$, which is a linear subspace of $X^{*}$, such that the identity embedding $J: Y \rightarrow X^{*}$ is compact and $\|J\| \leq 1$. Moreover,

$$
\left\{x_{1}^{*}, x_{2}^{*}, \ldots\right\} \subset J\left(B_{Y}\right) .
$$

Since $X$ has the weak $\mu$-bounded approximation property, for the operator $\left.J^{*}\right|_{X} \in$ $\mathcal{K}\left(X, Y^{*}\right)$, there exists a net $\left(S_{\alpha}\right) \subset \mathcal{F}(X, X)$ with $\left\|\left.J^{*}\right|_{X} S_{\alpha}\right\| \leq \mu$ for all $\alpha$ such that $S_{\alpha} \rightarrow I_{X}$ uniformly on compact subsets of $X$. Observe that $\left\|S_{\alpha}^{*} J\right\| \leq \mu$ for all $\alpha$ because $S_{\alpha}^{*} J=S_{\alpha}^{*}\left(\left.J^{*}\right|_{X}\right)^{*}=\left(\left.J^{*}\right|_{X} S_{\alpha}\right)^{*}$ and $\left\|\left.J^{*}\right|_{X} S_{\alpha}\right\| \leq \mu$. Using that $T_{\nu}\left(B_{E}\right)$ is a compact subset of $X$, let us fix $S_{\nu}:=S_{\alpha}$ such that

$$
\left\|\left.\left(S_{\nu} T_{\nu}-T_{\nu}\right)\right|_{E}\right\|=\sup \left\{\left\|S_{\nu} x-x\right\|: x \in T_{\nu}\left(B_{E}\right)\right\}<\varepsilon .
$$

Moreover, let $y_{n} \in B_{Y}$ satisfy $x_{n}^{*}=J y_{n}$. Then, for all $n$,

$$
\left\|S_{\nu}^{*} x_{n}^{*}\right\|=\left\|S_{\nu}^{*} J y_{n}\right\| \leq \mu\left\|y_{n}\right\| \leq \mu .
$$

Now we shall apply Lindenstrauss's compactness argument 13 to construct $f \in\left(X^{* *} \widehat{\otimes} X^{*}\right)^{*}$ in the following way. Observing that, for all $\nu=(E, F, G, \varepsilon)$ and for all $u \in B_{G}$,

$$
\begin{aligned}
\left|\operatorname{trace}\left(S_{\nu}^{* *} T_{\nu} u\right)\right| & =\left|\sum_{n=1}^{\infty} \lambda_{n}^{u}\left(T_{\nu} x_{n}^{* *}\right)\left(S_{\nu}^{*} x_{n}^{*}\right)\right| \\
& \leq \sum_{n=1}^{\infty}\left|\lambda_{n}^{u}\right|(\lambda+\varepsilon)\left\|S_{\nu}^{*} x_{n}^{*}\right\| \leq(1+\varepsilon)(\lambda+\varepsilon) \mu
\end{aligned}
$$

we consider, for all $u \in X^{* *} \widehat{\otimes} X^{*}$, the net $\left(u_{\nu}\right)_{\nu} \subset \mathbb{K}$ defined by

$$
u_{\nu}=\left\{\begin{array}{l}
\operatorname{trace}\left(S_{\nu}^{* *} T_{\nu} u\right), \quad u \in G \\
0, \quad \text { otherwise. }
\end{array}\right.
$$


Then, for all $\nu$,

$$
\left(u_{\nu}\right)_{u \in X^{* *} \widehat{\otimes} X^{*}} \in \prod_{u \in X^{* *} \widehat{\otimes} X^{*}}\left\{a \in \mathbb{K}:|a| \leq 2(\lambda+1) \mu\|u\|_{\pi}\right\} .
$$

Hence, by the compactness of the product space (Tychonoff's theorem is used here), $\left(\left(u_{\nu}\right)_{u \in X^{*} \hat{\otimes} X^{*}}\right)_{\nu}$ has a convergent subnet. Therefore, by passing to this pointwise convergent subnet, we may assume that, for all $u \in X^{* *} \widehat{\otimes} X^{*}$,

$$
\exists \lim _{\nu} u_{\nu} \in\left\{a \in \mathbb{K}:|a| \leq 2(\lambda+1) \mu\|u\|_{\pi}\right\} .
$$

Define $f: X^{* *} \widehat{\otimes} X^{*} \rightarrow \mathbb{K}$ by

$$
f(u)=\lim _{\nu} u_{\nu}, \quad u \in X^{* *} \widehat{\otimes} X^{*} .
$$

It is routine to verify that $f$ is linear and $\|f\| \leq \lambda \mu$. Thus $f \in\left(X^{* *} \widehat{\otimes} X^{*}\right)^{*}$.

Let $\varphi \in \mathcal{L}\left(X^{*}, X^{* * *}\right)$ be the operator that canonically corresponds to $f$. To show that $\varphi \in \overline{\mathcal{F}\left(X^{*}, X^{* * *}\right)}{ }^{w^{*}}$, we fix any $u_{k} \in X^{* *} \widehat{\otimes} X^{*}, k=1, \ldots, n$, and verify that, by passing to a subnet, $\left\langle T_{\nu}^{*} j_{X^{*}} S_{\nu}^{*}, u_{k}\right\rangle \rightarrow\left\langle\varphi, u_{k}\right\rangle$. In fact, considering $\nu=(E, F, G, \varepsilon)$ such that $u_{k} \in G, k=1, \ldots, n$, we have

$$
\left\langle T_{\nu}^{*} j_{X^{*}} S_{\nu}^{*}, u_{k}\right\rangle=\operatorname{trace}\left(S_{\nu}^{* *} T_{\nu} u_{k}\right)=u_{k \nu}, k=1, \ldots, n .
$$

Since $u_{k \nu} \rightarrow f\left(u_{k}\right)=\left\langle\varphi, u_{k}\right\rangle$, this implies the desired convergence.

To conclude the proof, let us show that, in fact, $j_{X^{*}}$ is the operator that canonically corresponds to $f$. For this end, since $\left(j_{X^{*}} x^{*}\right)\left(x^{* *}\right)=x^{* *}\left(x^{*}\right)$, we need to verify that

$$
f\left(x^{* *} \otimes x^{*}\right)=x^{* *}\left(x^{*}\right)
$$

for all $x^{*} \in X^{*}$ and $x^{* *} \in X^{* *}$. Let $\nu=(E, F, G, \varepsilon)$ be such that $x^{* *} \in E, x^{*} \in F$, and $x^{* *} \otimes x^{*} \in G$. Then $\left\|S_{\nu} T_{\nu} x^{* *}-T_{\nu} x^{* *}\right\|<\varepsilon\left\|x^{* *}\right\|$ and therefore

$$
\left|\operatorname{trace}\left(S_{\nu}^{* *} T_{\nu}\left(x^{* *} \otimes x^{*}\right)\right)-x^{* *}\left(x^{*}\right)\right|=\left|x^{*}\left(S_{\nu} T_{\nu} x^{* *}\right)-x^{*}\left(T_{\nu} x^{* *}\right)\right|<\varepsilon\left\|x^{* *}\right\|\left\|x^{*}\right\|,
$$

implying that $f\left(x^{* *} \otimes x^{*}\right)=x^{* *}\left(x^{*}\right)$ as desired.

\section{ACKNOWLEDGMENT}

The author thanks the referee for helpful suggestions that improved the exposition.

\section{REFERENCES}

[1] P.G. Casazza. Approximation properties. In: W.B. Johnson and J. Lindenstrauss (eds.) Handbook of the Geometry of Banach Spaces. Volume 1, Elsevier (2001) 271-316. MR1863695 (2003f:46012)

[2] W.J. Davis, T. Figiel, W.B. Johnson, and A. Peeczyński. Factoring weakly compact operators. J. Funct. Anal. 17 (1974) 311-327. MR0355536 (50:8010)

[3] J. Diestel and J.J. Uhl. Vector Measures. Mathematical Surveys 15. American Mathematical Society, Providence, Rhode Island, 1977. MR0453964 (56:12216)

[4] T. Figiel And W. B. Johnson. The approximation property does not imply the bounded approximation property, Proc. Amer. Math. Soc. 41 (1973) 197-200. MR0341032 (49:5782)

[5] G. Godefroy and P.D. Saphar. Duality in spaces of operators and smooth norms on Banach spaces. Illinois J. Math. 32 (1988) 672-695. MR955384 (89j:47026)

[6] A. Grothendieck. Produits tensoriels topologiques et espaces nucléaires. Mem. Amer. Math. Soc. 16 (1955). MR0075539(17:763c)

[7] W.B. Johnson. A complementary universal conjugate Banach space and its relation to the approximation problem. Israel J. Math. 13 (1972) 301-310. MR.0326356 (48:4700) 
[8] W.B. Johnson And T. OikhBerg. Separable lifting property and extensions of local reflexivity. Illinois J. Math. 45 (2001) 123-137. MR1849989(2002j:46014)

[9] W.B. Johnson, H.P. Rosenthal, And M. ZiPPIN. On bases, finite dimensional decompositions and weaker structures in Banach spaces. Israel J. Math. 9 (1971) 488-506. MR0280983 (43:6702)

[10] N.J. Kalton and D. Werner. Property (M), M-ideals, and almost isometric structure of Banach spaces. J. Reine Angew. Math. 461 (1995) 137-178. MR.1324212 (96m:46022)

[11] Å. Lima, O. NyGAARd, AND E. OJA. Isometric factorization of weakly compact operators and the approximation property. Israel J. Math. 119 (2000) 325-348. MR.1802659 (2002b:46031)

[12] Å. Lima And E. OJA. The weak metric approximation property. Math. Ann. 333 (2005) 471-484. MR2198796 (2006i:46025)

[13] J. Lindenstrauss. Extension of compact operators. Mem. Amer. Math. Soc. 48 (1964). MR0179580 (31:3828)

[14] J. Lindenstrauss and L. Tzafriri. Classical Banach Spaces I. Springer, Berlin-HeidelbergNew York, 1977. MR0500056 (58:17766)

[15] T. Oikhberg And H.P. Rosenthal. Extension properties for the space of compact operators. J. Funct. Anal. 179 (2001) 251-308. MR1809112 (2002b:47138)

[16] E. OJA. Geometry of Banach spaces having shrinking approximations of the identity. Trans. Amer. Math. Soc. 352 (2000) 2801-2823. MR1675226 (2000j:46034)

[17] E. OJA. The impact of the Radon-Nikodým property on the weak bounded approximation property. Rev. R. Acad. Cien. Serie A. Mat. 100 (2006) 325-331.

Faculty of Mathematics and Computer Science, Tartu University, J. Litvi 2, Ee-50409 TARTU, ESTONIA

E-mail address: eve.oja@ut.ee 